\title{
Escoamento uniforme em canais circulares lisos. Parte II: Comparação entre o método de Kazemipour e a equação de Manning ${ }^{1}$
}

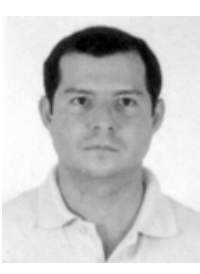

\author{
Maurício C. Goldfarb², Tarciso C. da Silva ${ }^{3}$, \\ Carlos A. V. de Azevedo ${ }^{4} \&$ Vera L. A. de Lima ${ }^{4}$
}

\begin{abstract}
1 Parte da Dissertação de Mestrado do primeiro autor apresentada na UFCG
2 DTCC/CT/UFPB. CEP 58059-900, João Pessoa, PB. Fone (83) 216-7037. E-mail: goldfarb@Irh.ct.ufpb.br (Foto)

${ }^{3}$ DTCC/CT/UFPB. Fone (83) 216-7684. E-mail: tarciso@Irh.ct.ufpb.br

${ }^{4}$ DEAg/CCT/UFCG. Av. Aprígio Veloso, 882, Bodocongó, CEP 58109-970, Campina Grande, PB. Fone: (83) $310-1056$.

E-mail: cazevedo@deag.ufcg.edu.br, antunes@deag.ufcg.edu.br
\end{abstract}

Protocolo $44-6 / 3 / 2003$ - Aprovado em 30/4/2004

\begin{abstract}
Resumo: A equação de Chezy-Manning e diversas outras utilizadas no cálculo do escoamento uniforme em canais, não representam satisfatoriamente o efeito da forma da seção transversal do escoamento, o que geralmente conduz a resultados de pouca precisão. Uma metodologia desenvolvida por Kazemipour \& Apelt (1980) a partir da equação de von Karman Prandtl para tubos pressurizados, denominada método de Kazemipour, tem-se mostrado bastante eficiente no cálculo do escoamento em canais circulares lisos. Neste trabalho, o método de Kazemipour, ajustado numericamente, foi confrontado com a equação de Manning. Utilizaram-se, na análise, valores de vazão calculados pelas duas metodologias e dados experimentais de confiabilidade. De acordo com os parâmetros avaliados, o método de Kazemipour mostrou-se superior à equação de Manning para cálculo do escoamento em canais circulares lisos.
\end{abstract}

Palavras-chave: irrigação, vazão, hidráulica

\section{Uniform flow in smooth circular channels. Part II: Comparison between the Kazemipour method and the Manning equation}

\begin{abstract}
The Chezy-Manning equation as well as several other equations used in the calculation of the uniform flow in channels does not represent satisfactorily the shape effect of the flow crosssectional area, which results in little precision. A methodology developed by Kazemipour \& Apelt (1980), considering the von Karmam Prandtl equation for pressurized tubes, denominated as method of Kazemipour, has showin quite efficient in the calculation of the flow in smooth circular channels. In this research, the method of Kazemipour, numerically adjusted was confronted with the Manning equation. In the analysis, discharge values calculated by the two methodologies and reliable experimental data were used. According to the evaluated parameters, the method of Kazemipour showed to be superior to Manning's equation for flow calculation in smooth circular channels.
\end{abstract}

Key words: irrigation, discharge, hydraulics

\section{INTRODUÇÃO}

A partir de estudos teóricos e experimentais, o engenheiro francês Antoine Chezy desenvolveu, em 1769, uma equação, para cálculo do escoamento tanto em tubulações pressurizadas como em canais abertos. Na equação proposta por Chezy, os parâmetros do escoamento eram relacionados através da constante experimental C, denominado coeficiente de Chezy.

Em 1889, Manning determinou o valor do coeficiente $\mathrm{C}$ da equação de Chezy para o cálculo do escoamento uniforme livre em canais, como sendo igual à razão entre o raio hidráulico elevado à potência de $1 / 6$ e o parâmetro $n$, representativo da rugosidade das paredes do canal. Atualmente, a equação de Manning é uma das metodologias de cálculo mais utilizadas por projetistas de países ocidentais no dimensionamento de canais abertos. Na maneira como é apresentada, esta equação pode ser utilizada no cálculo do escoamento uniforme em canais, qualquer que seja a forma geométrica da seção transversal.

De acordo com Silva (1992), as diversas formulações matemáticas apresentadas para o cálculo do escoamento uniforme em canais, a exemplo da própria equação de Manning, representam a geometria da seção transversal simplesmente pela 
área A e o raio hidráulico $\mathrm{R}$. A suposição de que o raio hidráulico e a área da seção transversal sejam suficientes para representar a forma da seção transversal, foi comprovada como não verdadeira, nas conclusões de Shih \& Grigg (1967). A esta consideração simplista está associada a baixa eficiência de diversas equações utilizadas para o cálculo da resistência ao escoamento uniforme em canais abertos. Estudos experimentais demonstraram que a equação de Manning pode ocasionar erro de até 30\% em seus resultados (WPCF,1972).

Buscando solucionar, de maneira mais eficiente, o cálculo do escoamento nos canais circulares, Kazemipour \& Apelt (1980) desenvolveram uma metodologia de cálculo, denominada método de Kazemipour, a qual tem sido comprovada por diversos autores, a exemplo Silva (1992), Nalluri \& Adepoju (1985) entre outros, como uma metodologia de cálculo de bastante eficiência. Este procedimento leva em conta os efeitos da forma da seção transversal do escoamento uniforme através de dois parâmetros adimensionais.

Desta forma, o objetivo deste trabalho foi comparar as eficiências do método de Kazemipour e da equação de Manning, no cálculo do escoamento uniforme, em canais circulares lisos.

\section{MATERIAL E MÉTODOS}

Neste trabalho, o método de Kazemipour é aplicado a partir do ajuste numérico desenvolvido de acordo com o procedimento proposto por Silva \& Figueiredo (1993).

A comparação entre o método de Kazemipour e a equação de Manning, foi feita através de dados de vazões gerados pelas duas metodologias e de dados de laboratório obtidos por Kazemipour \& Apelt (1980), aceitos por diversos autores, a exemplo de Nalluri \& Adepoju (1985), Silva (1992) entre outros, como dados experimentais de excelente qualidade.

\section{O Método de Kazemipour ajustado}

A utilização do método de Kazemipour para cálculo do escamento em canais circulares, conforme apresentada por Kazemipour \& Apelt (1980), é feita com base no parâmetro adimensional, $\psi$, que representa a forma da geometria da seção transversal do escoamento. O parâmetro $\psi$, por sua vez, é composto da razão entre dois outros parâmetros adimensionais, denominados primeiro e segundo parâmetros de forma do método de Kazemipour. A equação seguinte expressa a relação entre tais parâmetros.

$$
\psi=\psi_{1} / \psi_{2}
$$

O primeiro parâmetro de forma do método de Kazemipour, $\psi_{1}$, pode ser calculado a partir dos dados da geometria do escoamento. A Eq. (2) apresenta a expressão de cálculo deste parâmetro.

$$
\psi_{1}=(\mathrm{P} / \mathrm{D})^{1 / 2}
$$

donde P é o perímetro molhado do escoamento e $\mathrm{D}$ o diâmetro interno do canal. A Figura 1 sintetiza a simbologia das grandezas utilizadas neste trabalho, enquanto a profundidade efetiva do escoamento, $\mathrm{y}_{\mathrm{D}}$, pode ser obtida através da razão entre a área molhada A e o diâmetro estrutural D, nela apresentado.

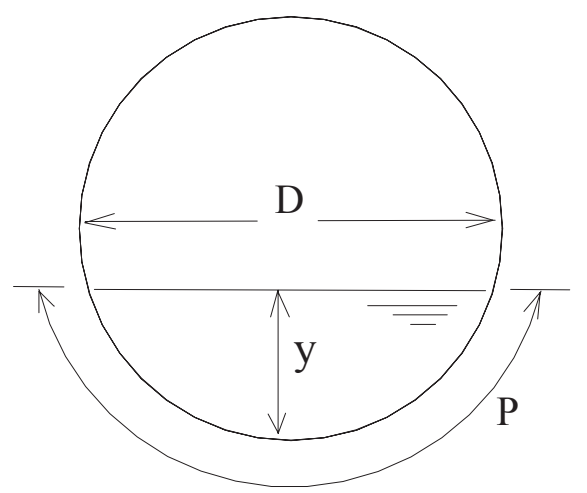

Figura 1. Grandezas geométricas utilizadas

O segundo parâmetro de forma do método de Kazemipour, $\psi_{2}$, função do diâmetro interno do canal, D, e da profundidade efetiva do escoamento, $\mathrm{y}_{\mathrm{D}}$, pode ser calculado através da Eq. (3), obtida por Goldfarb (2002) de acordo com o procedimento desenvolvido por Silva \& Figueiredo (1993).

$$
\psi_{2}=1,864 \exp \left\{0,084\left[\ln \left(\mathrm{D} / \mathrm{y}_{\mathrm{D}}\right)^{2}\right]-0,589\left[\ln \left(\mathrm{D} / \mathrm{y}_{\mathrm{D}}\right)\right]\right\}
$$

De posse do parâmetro de forma $\psi$, o fator de atrito foi determinado para escoamento uniforme em canais de seção circular, utilizando-se as equações do coeficiente de atrito para tubos pressurizados, conforme metodologia apresentada por Kazemipour \& Apelt (1980). A Eq. 4 apresenta a expressão para o fator de atrito para canais.

$$
\mathrm{fc}=\psi \cdot f
$$

em que fé o coeficiente de atrito para tubulações pressurizadas e fc é o coeficiente de atrito para canais de seção circular.

Uma vez determinado o coeficiente de atrito fc para escoamento uniforme em canais, a velocidade média do escoamento foi finalmente determinada utilizando-se a Eq. 5, derivada da equação racional para cálculo do escoamento em tubulações pressurizadas.

$$
V=\sqrt{\frac{8 g R S}{f_{c}}}
$$

sendo $\mathrm{V}$ a velocidade média do escoamento no canal, g a aceleração da gravidade, $\mathrm{R}$ o raio hidráulico, $\mathrm{S}$ a declividade do fundo do canal, e fc o fator de atrito para canais lisos.

\section{A equação de Manning}

A seguir apresenta-se a formulação da Equação de Manning utilizada nesta pesquisa.

$$
Q=\frac{A}{n} R^{2 / 3} S^{1 / 2}
$$


sendo A a área da seção transversal do escoamento, $\mathrm{R}$ o raio hidráulico, $\mathrm{S}$ a declividade do fundo do canal e n o coeficiente de rugosidade de Manning que, apesar de representar a rugosidade das paredes do canal, é considerado um fator adimensional (Raju, 1981).

A formulação de Manning, conforme apresentada na Eq. (6), é a mais utilizada por engenheiros no dimensionamento de canais, e o coeficiente $\mathrm{n}$ é facilmente encontrado tabelado para diversos tipos de materiais de constituição das paredes e do fundo do canal, em compêndios da literatura especializada.

\section{RESULTADOS E DISCUSSÃO}

A comparação entre o método de Kazemipour e a equação de Manning se dá através da vazão calculada pelas duas metodologias, tomando-se como referência valores experimentais de escoamento em canais circulares lisos. Os dados experimentais utilizados foram submetidos a críticas e análise de consistência por autores, a exemplo de Nalluri \& Adepoju (1985) entre outros, sendo aceitos como resultados experimentais de excelente qualidade, o que justifica sua adoção como dados de referência na análise proposta.

No método de Kazemipour ajustado numericamente por Goldfarb (2002), as vazões foram calculadas de acordo com a seqüência de cálculo proposta por Silva (1992), que torna o procedimento solucionável a partir de equações explícitas. Neste caso, o segundo parâmetro adimensional do método de Kazemipour foi obtido diretamente da Eq. (3) sem a necessidade de recursos gráficos.

Nas vazões calculadas através da equação de Manning, o valor utilizado para o coeficiente $\mathrm{n}$, que representa a rugosidade das paredes do canal, foi o que proporcionou o melhor resultado para os valores calculados através desta equação, caso em que o valor $\mathrm{n}=0,0098$ foi o que resultou nos valores de vazões calculadas mais próximo dos experimentais, utilizados como referência. Na literatura especializada não se dispõe de valores do coeficiente $\mathrm{n}$ de Manning com este grau de precisão, o que conduziria a resultados práticos, porém menos precisos desta equação. Azevedo Neto (1998) indica para canais lisos conduzindo água limpa, o valor de $\mathrm{n}=0,012$.

A Figura 2 apresenta uma relação entre as vazões observadas experimentalmente, variando de 3,6 a 49,5 L s'-1, e os valores calculados através da equação de Manning e do método de Kazemipour ajustado numericamente. Para tanto, cada ponto experimental é representado através da altura hidráulica, do diâmetro interno do canal, da temperatura da água e da declividade do fundo do canal. Nesta figura, a reta diagonal representa o lugar geométrico ideal dos pontos calculados, ou seja, quando o valor calculado seria idêntico ao valor de referência obtido experimentalmente. Observa-se que para valores de vazão menores que $10 \mathrm{~L} \mathrm{~s}^{-1}$, existe relativa equivalência na eficiência das duas metodologias, porém para valores superiores a $10 \mathrm{~L} \mathrm{~s}^{-1}$, o método de Kazemipour apresenta-se notadamente superior à equação de Manning, visto que seus resultados estão mais próximos da reta diagonal, ou seja, dos valores de referência.

Na Figura 3 constatou-se diferença significativa na dispersão dos pontos gerados, tanto pela equação de Manning como

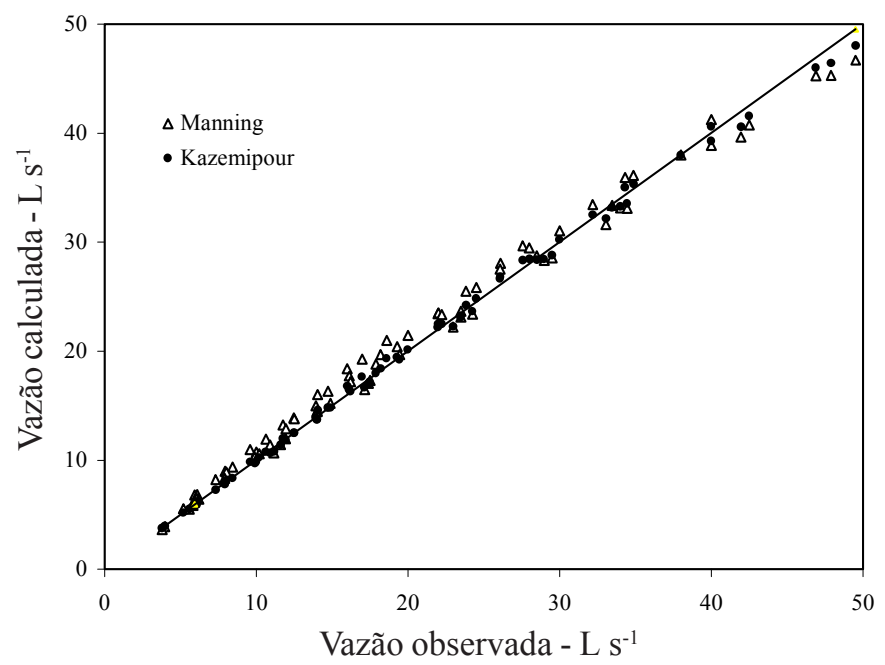

Figura 2. Relação entre as vazões observadas experimentalmente e as calculadas através da equação de Manning e do método de Kazemipour

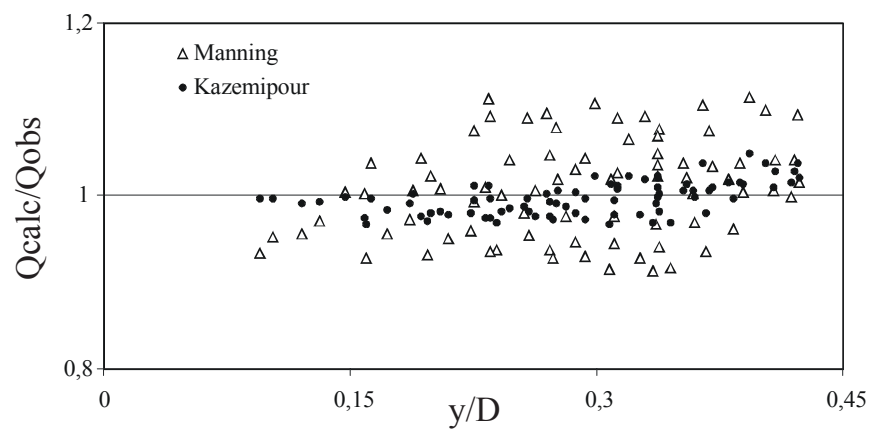

Figura 3. Vazões adimensionalizadas em função do parâmetro geométrico $\mathrm{y} / \mathrm{D}$

através do método de Kazemipour ajustado numericamente, com o aumento da altura hidráulica do escoamento. As duas metodologias tenderam a superestimar a vazão calculada quando a altura hidráulica se aproxima do valor igual à metade do diâmetro interno do canal. No caso do método de Kazemipour, esta tendência já foi comprovada através dos estudos feitos por Nalluri \& Adepoju (1985).

De maneira geral, pouca concordância observada nos resultados da equação de Manning, quando comparados aos valores calculados através do método de Kazemipour, está associada à insuficiente representação da geometria da seção transversal do escoamento por esta equação. A equação de Manning representa a geometria da seção transversal simplesmente pela área $\mathrm{A}$ e o raio hidráulico $\mathrm{R}$, que não são parâmetros, por si sós, capazes de representar o fenômeno conhecido como efeitos de forma, estudados por diversos pesquisadores, a exemplo de Kirkgoz \& Ardiçhoglu (1997), Silva (1992), Oliveira (1994), Kazemipour \& Apelt (1980), Replogle \& Chow (1966) entre outros.

\section{CONCLUSÕES}

1. O desempenho do método de Kazemipour mostrou-se superior ao da equação de Manning, para o cálculo do 
escoamento uniforme em canais circulares lisos, em todas as razões de aspecto avaliadas.

2. As vantagens da equação de Manning, quando comparada com o método de Kazemipour, se dá devido à sua facilidade de uso, já que se trata de uma equação monômica; no entanto, utilizando-se um computador ou uma calculadora programável, o método de Kazemipour pode ser facilmente aplicável.

\section{AGRADECIMENTOS}

Ao Professor Dr. Colin J. Apelt, da Universidade de Queensland (Austrália), que gentilmente forneceu dados experimentais imprescindíveis à elaboração deste trabalho, e à Coordenação de Aperfeiçoamento de Pessoal de Nível Superior (CAPES) pela concessão de bolsa de estudo ao primeiro autor.

\section{LITERATURA CITADA}

Azevedo Netto, J.M.; Fernandez y Fernandez, M.; Araújo, R.; Eiji Ito, A. Manual de hidráulica. 8. ed. São Paulo: Editora Edgard Blücher Ltda, 1998. 669p.

Goldfarb M.C. Resistência ao escoamento uniforme em canais circulares lisos, Campina Grande: Universidade Federal da Paraíba, 2002. 68p. Dissertação Mestrado

Kazemipour, A.K.; Apelt, C.J. Shape effects on resistance to smooth semi-circular channels. Brisbane: Department of Civil Engineering, University of Queensland, 1980. 26p. Research Report No. CE18
Kirkgoz, M.S.; Ardiçhoglu, M. Velocity profiles of developing and developed open channel flow. Journal of the Hydraulics Engineering, Delft, Netherlands, v. 123, n.12, p. 1099-1105, 1997.

Nalluri, C.; Adepoju, B.A. Shape effects on resistance to flow in smooth channels of circular cross-section. Journal of Hydraulic Research, Delft, Netherlands, v. 23, n.1, p.25-36, 1985.

Oliveira, J.M.S. Resistência ao escoamento uniforme em canais triangulares lisos, João Pessoa: Universidade Federal da Paraíba, 1994. 66p. Dissertação Mestrado

Raju, K.G.R. Flow through open channels. 1. ed. New Delhi: McGraw-Hill Publishing Company Limited, 1981. 330p.

Replogle, J.A; Chow, V.T. Tractive-force distribution in open channels. Journal of the Hydraulics Division, Delft, Netherlands, v.92, n. HY2, p.169-191, 1966.

Shih C.C.; Grigg, N.S. A reconsideration of the hydraulic radius as a geometric quantity in open channel hydraulics. In: Proceeding of the 12th Congress. Fort Collins, Colorado, USA: I.A.R.H., v.1, (Paper A36), 1967. p.288-296.

Silva, T.C. Efeitos de forma na resistência ao escoamento uniforme em canais, São Paulo: Escola Politécnica da Universidade de São Paulo, 1992. 85p. Tese Doutorado

Silva, T.C.; Figueiredo P.H.S. Ajuste polinomial: três estudos de casos utilizando variáveis transformadas In: Congresso Ibero Latino-Americano de Métodos Computacionais em Engenharia, 14, 1993, São Paulo. Anais... 1993.p.1212-1219

WPCF - Water Pollution Control Federation. Design and construction of sanitary and storm sewers. Manual of Practice, 2 ed. 1972. Washington, D.C. 347p. 